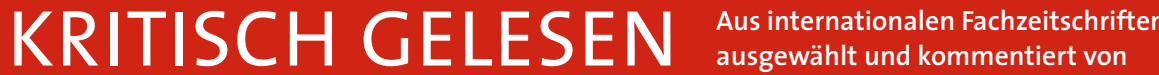

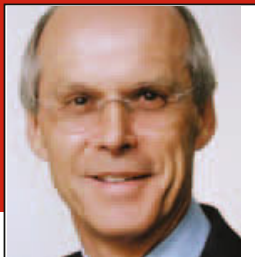

Prof. Dr. med. H. S. FüeßI

Isar-AmperKlinikum, $\mathrm{KI}$. MünchenOst, Haar

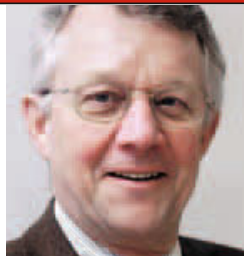

Prof. Dr. med. H. Holzgreve Internist, Kardiologische Praxis, München

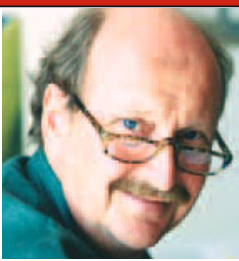

Prof. Dr. med. E. Ernst

Peninsular

Medical School,

University of Exeter/UK

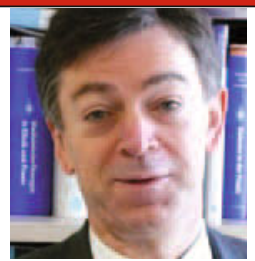

Prof. Dr. med. H. Hauner

Ernährungsmedizin, TU München

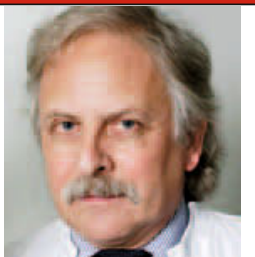

Prof. Dr. med. H.-C. Diener

Neurologie, Universität Duisburg-Essen

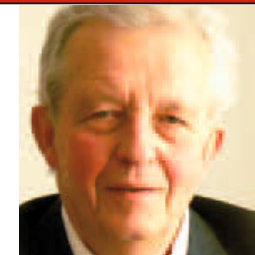

Prof. Dr. med. K. Malberg Immunologie, DresdenLoschwitz

\section{Viele NSAID sind nicht gut für Herz und Hirn}

\begin{abstract}
Wer einem Patienten ein selektives oder nicht selektives, nicht steroidales Antiphlogistikum (NSAID) verordnet, muss mit einem erhöhten kardiovaskulären Risiko rechnen.
\end{abstract}

— In einer Netzwerkanalyse werden alle randomisierten Studien mit Substanzen einer bestimmten Arzneimittelgruppe gegen Placebo oder untereinander gemeinsam ausgewertet. Die Autoren fanden 31 Studien, die ein NSAID gegen Placebo oder gegen ein oder mehrere andere NSAID's über wenigstens 100 Patientenjahre verglichen und 116429 Patienten mit 117218 Patientenjahre einschlossen. Eine weitere Voraussetzung war, dass in den einzelnen Studien mindestens zehn Herzinfarkte berichtet wurden. Insgesamt liegen Daten für sieben verschiedene NSAID's vor. Die umfangreichsten Daten gibt es zu Etoricoxib und Diclofenac.

Das Herzinfarktrisiko ist gegenüber Placebo am stärksten erhöht unter Rofecoxib (2,12-fach) und Lumiracoxib $(2,00)$, also unter den beiden Substanzen, die in Deutschland nicht zugelassen sind (s. Tab.). Die Zahlen variieren bei den einzelnen Endpunkten: Am ungünstigsten schneiden bei den Schlaganfällen Ibuprofen $(3,36)$ und Diclofenac $(3,98)$, bei kardiovaskulären Todesfällen Etoricoxib $(4,07)$ und Diclofenac $(3,98)$, bei der Gesamtmortalität Diclofenac $(2,31)$ und Etoricoxib

\section{Tabelle 1}

Risikoerhöhung für kardiovaskuläre Komplikationen durch NSAID im Vergleich zu Placebo

\begin{tabular}{l|c|c|c|c|} 
NSAID & Herzinfarkt & Schlaganfall & CV Tod & Komb. Endpunkt \\
\hline Naproxen & 0,82 & 1,76 & 0,98 & 1,22 \\
\hline Ibuprofen & 1,61 & $3,36^{*}$ & 2,39 & $2,26^{*}$ \\
\hline Diclofenac & 0,82 & $2,86^{*}$ & $3,98^{*}$ & 1,60 \\
\hline Celecoxib & 1,35 & 1,12 & 2,07 & 1,43 \\
\hline Etoricoxib & 0,75 & 2,67 & $4,07^{*}$ & 1,53 \\
\hline Rofecoxib & $2,12^{*}$ & 1,07 & 1,58 & $1,44^{*}$ \\
\hline $\begin{array}{l}\text { Lumiracoxib } \\
\text { (CV = kardiovaskulär; }{ }^{*}=\text { signifikant) }\end{array}$ & 2,00 & 1,89 & $2,04^{*}$ \\
\hline
\end{tabular}

$(2,29)$ und bei einem kombinierten Endpunkt Ibuprofen $(2,26)$ und Lumiracoxib $(2,04) \mathrm{ab}$.

\section{Kommentar}

(1) Das Risiko liegt bei allen Substanzen und allen Endpunkten über 1,o, d.h. es ist bei allen Substanzen erhöht, ausgenommen für Naproxen bei Herzinfarkten $(0,82)$ und kardiovaskulären Todesfällen $(0,98)$ sowie bei Diclofenac und Etoricoxib bei Herzinfarkten (0,82 bzw. 0,75).

(2) Das Risiko ist für einzelne Substanzen nicht über alle Endpunkte gleichmäßig verteilt, z.B. bei Diclofenac und Etoricoxib für Herzinfarkte $(0,82$ bzw. 0,75) und für kardiovaskuläre Todesfälle (3,98 bzw. 4,07).

(3) Naproxen schneidet insgesamt am besten $a b$.

(4) Das absolute Risiko für kardiovaskuläre Komplikationen ist zwar gering, doch werden viele Millionen Patienten mit NSAID behandelt. Diese Arzneimittelgruppe ist nicht ideal für die langfristige, symptomatische Behandlung muskuloskelettaler Beschwerden.

H. HOLZGREVE

\footnotetext{
- S. Trelle et al.

Cardiovascular safety of non-steroidal antiinflammatory drugs: network meta-analysis. Brit. Med. J. doi: 10.1136/bmj.c7086
} 